\title{
FLITECAM: early commissioning results
}

\author{
Sarah E. Logsdon*a, Ian S. McLean ${ }^{a}$, Eric E. Becklin ${ }^{\mathrm{b}}$, Edward W. Dunham ${ }^{\mathrm{c}}$, Ryan T. Hamilton ${ }^{\mathrm{b}}$, \\ Christopher A. Johnson ${ }^{a}$, Jennifer W. Milburn ${ }^{d}$, Maureen L. Savage ${ }^{b}, S^{2}$, Rindev S. Shenoy ${ }^{b}$, \\ Erin C. Smith ${ }^{\mathrm{e}}$, William D. Vacca ${ }^{\mathrm{b}}$ \\ a University of California, Los Angeles, CA, USA 90095-1547; ' USRA, NASA Ames Research \\ Center, Moffett Field, CA, USA 94035; ${ }^{\circ}$ Lowell Observatory, Flagstaff, AZ, USA 86001; \\ ${ }^{\mathrm{d}}$ California Institute of Technology, Pasadena, CA, USA 91125; ${ }^{\mathrm{e}}$ NASA Ames Research Center, \\ Moffett Field, CA, USA 94035
}

\begin{abstract}
We present a status report and early commissioning results for FLITECAM, the 1-5 micron imager and spectrometer for SOFIA (the Stratospheric Observatory for Infrared Astronomy). In February 2014 we completed six flights with FLITECAM mounted in the FLIPO configuration, a co-mounting of FLITECAM and HIPO (High-speed Imaging Photometer for Occultations; PI Edward W. Dunham, Lowell Observatory). During these flights, the FLITECAM modes from $\sim 1-4 \mu \mathrm{m}$ were characterized. Since observatory verification flights in 2011, several improvements have been made to the FLITECAM system, including the elimination of a light leak in the FLITECAM filter wheel enclosure, and updates to the observing software. We discuss both the improvements to the FLITECAM system and the results from the commissioning flights, including updated sensitivity measurements. Finally, we discuss the utility of FLITECAM in the FLIPO configuration for targeting exoplanet transits.
\end{abstract}

Keywords: commissioning, spectrometer, near-infrared, SOFIA, airborne astronomy

\section{INTRODUCTION}

FLITECAM is a near-infrared $(1-5 \mu \mathrm{m})$ camera and grism spectrometer for NASA's Stratospheric Observatory for Infrared Astronomy (SOFIA). FLITECAM was developed by principal investigator Ian McLean, at the UCLA Infrared Laboratory. FLITECAM's optical design and ground-based performance have been described in many earlier papers ${ }^{1,2,3}$. The basic instrument properties are as follows: the optics accept a circular field of view of $\sim 8^{\prime}$ in diameter, which is inscribed within a 1024 × 1024 pixel InSb Aladdin III detector from Raytheon. The image scale on SOFIA is 0.475 arcsec/pixel. There are two primary observing modes, direct imaging and grism spectroscopy. Two filter wheels house a suite of nine broad and narrow-band imaging filters, a set of specialized order sorting filters for spectroscopy, and three direct-ruled KRS-5 grisms. A mechanism allows a slit mask to be inserted into the focal plane for spectroscopy. FLITECAM also has a rapid-exposure "movie" mode for occultation studies, as well as a pupil-viewing mode for observations near $3.5 \mu \mathrm{m}$. Because FLITECAM's liquid cryogenic cooling system was designed to operate in either a vertical or horizontal orientation, the instrument could be and was tested at the Cassegrain focus of the Lick Observatory Shane telescope $(3.0 \mathrm{~m}, \mathrm{f} / 17)$ prior to installation at the Nasmyth focus of the SOFIA telescope $(2.5 \mathrm{~m}, \mathrm{f} / 19.7)$. FLITECAM was deployed to Lick Observatory on eight separate occasions over many years. Observations made during those runs resulted in three scientific papers ${ }^{4,5,6}$ plus several conference papers, and two successful Ph.D. theses (Amanda K. Mainzer and Erin C. Smith).

During the summer and fall of 2011, FLITECAM was delivered to SOFIA and co-mounted with HIPO, the High-speed Imaging Photometer for Occultations ${ }^{7,8}$ (PI Edward Dunham, Lowell Observatory). HIPO is mounted on-axis and FLITECAM mounts above it. A periscope composed of a dichroic beam-splitter and fold-mirror in front of HIPO reflects infrared light to FLITECAM. This combination (known as FLIPO) was used during line operations and four observatory verification flights in October $2011^{9}$.

*slogsdon@astro.ucla.edu

Ground-based and Airborne Instrumentation for Astronomy V, edited by

Suzanne K. Ramsay, lan S. McLean, Hideki Takami, Proc. of SPIE Vol. 9147,

$91472 Y$ - (c) 2014 SPIE - CCC code: $0277-786 X / 14 / \$ 18 \cdot$ doi: $10.1117 / 12.2056874$ 
Commissioning of FLITECAM in the FLIPO configuration was not performed on those 2011 flights because the Observatory itself was still under development. There were two attempts to begin FLIPO commissioning in the spring and fall of 2013, but these flights were postponed or curtailed for a variety of reasons. Finally, six very successful commissioning flights were undertaken in February 2014. In this paper we present a brief update on FLITECAM's properties and we discuss the early results from the 2014 commissioning flights in FLIPO configuration.

\section{INSTRUMENT STATUS AND UPDATES}

Several improvements have been made to FLITECAM following the 2011 observatory verification flights. For example, overheating of the FLITECAM electronics system just prior to the verification flights in 2011 resulted in a missing detector quadrant during the flight series. After the flight series was completed, the problem of the missing detector quadrant was traced to a faulty board in the digital signal processing electronics. The problem was corrected with a spare board, and all four quadrants of the detector became fully functional again.

During that same time period, images taken with our narrow band filter set, located in the second filter wheel, revealed a light-leak, or glint. This glint seemed to disappear when a blocking filter was used in the first filter wheel. The glint was traced to a long-wavelength light leak through an oversized aperture in the interface plate between the two filter wheels. When the aperture between the wheels was sized correctly for the optical beam, the glint was eliminated, without the use of a blocking filter in the first wheel.

As we prepared for FLITECAM's first flights on SOFIA, we noticed that the hold time for liquid helium in the cryostat had deteriorated from the $\sim 15$ hours that had been achieved when FLITECAM was used at Lick Observatory. Several changes had been made to the cryogenic venting system to meet flight safety standards, including the addition of a cluster of burst disks and pressure relief devices. At one point in 2013, the hold time had dropped to $\sim 6$ hours, which is less than the normal flight time of 9 hours. This behavior was finally tracked down to thermo-acoustic oscillations in the liquid helium vent system. Oscillations were driven by the opening and closing of a 1.4 psi mechanical pressure relief device added as part of the safety system. When a variable pressure relief device, essentially a classical rubber hose closed at one end and with a narrow slit cut into its side, was used to permit continuous venting of helium gas without building up pressure, the oscillations were significantly reduced, and the hold time returned to $\sim 15$ hours, more than adequate to keep the detector cold for an entire flight.

In early 2014 , the instrument control computer which runs all of the observing control software was upgraded from a Dell PowerEdge 750 Server to an Apple Mac Mini, which was co-mounted with another Mac Mini cold spare. Several improvements were also made to the control software itself, including updates to the Astronomical Observing Request (AOR) software. One important change to the AOR software is the ability to accept a dither-nod-dither pattern sequence. This change allows the observer to execute a dither sequence on a target, nod to a second field, and execute another dither sequence, all while maintaining guiding on either one or two guide stars (depending on the size of the nod). The dither-nod-dither sequence is ideal for extended objects because the second dither pattern could be some distance away in order to produce a clean sky frame for flat fielding.

Another notable software update was the development of a continuous data-taking mode. With one click of a button, the observer can execute as many repetitions of a single exposure as necessary. This feature is particularly useful for high cadence data observed over long observing legs, such as transits (see Section 3.5). Other more minor software changes included things such as header keyword updates for Data Cycle System (DCS) ingestion.

For the commissioning flights in February 2014, FLITECAM was once again co-mounted with HIPO in the FLIPO configuration as shown in Figure 1. As noted above, this arrangement includes a dichroic beam-splitter and fold-mirror in front of the FLITECAM entrance window. Since the time of the FLIPO flights in 2011, the dichroic beamsplitter has received a new and improved coating, with higher IR reflectivity ${ }^{10}$. The IR reflection now rises rapidly from $\sim 80 \%$ at $1.25 \mu \mathrm{m}$ to over $95 \%$ beyond $3 \mu \mathrm{m}$. Currently, these components remain relatively warm during flight, and therefore FLITECAM is exposed to an increased background, which limits the useful wavelength range to about 1-4 $\mu \mathrm{m}$ in the FLIPO configuration. In principle, it may be possible in the future to operate a cooling fan that will enable the FLIPO fore-optics to cool down to the temperature of the telescope. 


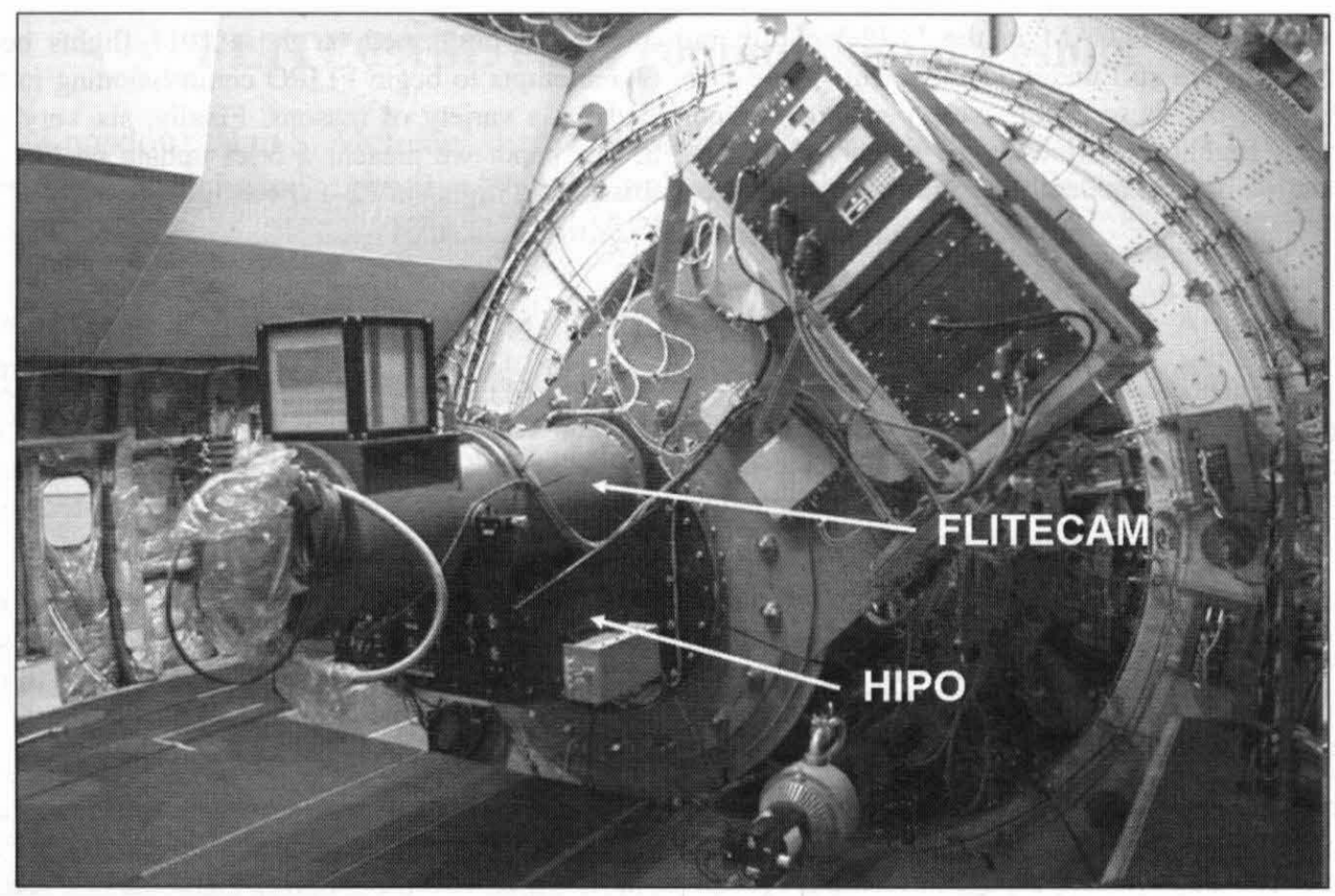

Figure 1. FLITECAM (blue cylinder) co-mounted on the SOFIA with HIPO (black). Upper right: FLITECAM electronics.

FLITECAM's specifications are essentially unchanged. In imaging mode, the scale is $0.475^{\prime \prime}$ per pixel, and the field of view on the $1024 \times 1024 \mathrm{InSb}$ detector is approximately 8 minutes of arc in diameter. FLITECAM is cooled with liquid nitrogen and liquid helium; the Aladdin III InSb detector is operated at $30 \mathrm{~K}$. The detector is an engineering grade device. It exhibits large variations in quantum efficiency, by almost a factor of two, across the array. There are also bad regions in the corners. Nevertheless, the non-uniform response flat-fields out quite well. Optical image quality degrades towards the outer edge of the large field of view due to coma and flare. The FLITECAM imaging pipeline uses the enclosed 5.6' x 5.6' area for best image quality, and to be consistent with dither pattern executions. As mentioned in the introduction, there are two filter wheels. The first wheel holds the standard JHKLL'M filters and a set of custom ordersorting filters for grism spectroscopy. The second wheel holds three KRS-5, direct-ruled, grisms, each with different groove densities. In addition, the second wheel holds six narrow band filters designated, Paschen- $\alpha$, Paschen- $\alpha$ continuum, $3.08 \mu \mathrm{m}$ Ice, PAH, narrow band $\mathrm{L}(\mathrm{nbL})$, and narrow band $\mathrm{M}(\mathrm{nbM})$. A slide mechanism in the cold focal plane carries a slit mask into the beam for spectroscopy mode. The slit mask contains two slits that are both $60^{\prime \prime}$ long and contiguous with one another. The wide slit ( $\sim 4$ pixels) gives a resolving power of $\sim 1,300$, and the narrower slit $(\sim 2$ pixels) gives a resolving power of $\sim 2,000$. Each of the three KRS- 5 grisms can be used in three orders, resulting in nine possible spectroscopic settings. Spectra are dispersed vertically on the detector display. Finally, there is also an optional pupil viewing lens which can be moved into the beam. FLITECAM's instrument specifications are given on the SOFIA website (http://sofia.usra.edu/), and in the referenced papers. In the FLIPO configuration, the $\mathrm{L}, \mathrm{L}^{\prime}, \mathrm{M}$, and narrow band $\mathrm{M}(\mathrm{nbM})$ filters are not used.

\section{RESULTS FROM COMMISSIONING FLIGHTS}

As already mentioned, FLITECAM was operated in the FLIPO configuration for six flights on SOFIA in February 2014. These flights, \#144-149, had multiple goals. Of highest priority was the commissioning of the instrument. Several flight legs were dedicated to boresight tests, shear-layer tests, guiding/tracking tests, dither pattern tests, nodding and offsetting tests, and image quality tests. However, several guest investigator programs were also carried out under shared risk conditions, and Director's discretionary time was invoked to observe a Target of Opportunity - the Type Ia supernova, SN2014J, in M82. In this section, we discuss the preliminary results of commissioning tests, and we highlight some of the exciting scientific potential of FLITECAM. 


\subsection{Image quality, zeropoints and filter profiles}

The telescope does not have a pre-flight cooling system, so the telescope cools rapidly during the early part of the flight. An algorithm has been developed to estimate the focus position of the secondary mirror as a function of temperature, and the focus is updated by the telescope operator throughout the flight. Our best images had a FWHM of about 6 pixels $\left(\sim 2.85^{\prime \prime}\right)$, but $3^{\prime \prime}$ was more typical, and there was often a slight elongation in the cross-elevation axis. It is noteworthy that FLITECAM produced seeing-limited images $\sim 1.2^{\prime \prime}$ during observations at Lick Observatory. Turbulence, cavity seeing, defocus and telescope jitter are likely to be the main factors affecting image quality.

Zeropoint (ZP) magnitudes, defined as the magnitude corresponding to a measured signal of $1 \mathrm{DN} / \mathrm{s}$, were obtained for most of FLITECAM's filters in the 1-4 $\mu \mathrm{m}$ range. Two standard stars that have been fully modeled in the near infrared ${ }^{11}$ were used for the determination of the zeropoints. The final zeropoint for each band is the weighted mean of the individual measurements, typically five frames per star, using the 2MASS magnitude as a reference. Table 1 below shows the results for the J, H, and $\mathrm{K}$ filters. Also tabulated are zeropoints for the pair of $1 \%$ wide $(\Delta \lambda / \lambda)$ filters associated with Paschen- $\alpha(1.875 \mu \mathrm{m})$ and its adjacent continuum at $1.90 \mu \mathrm{m}$. An average of the 2MASS H and Ks magnitudes was used as a reference for these special filters. The third column of Table 1 gives the conversion factor from measured counts per second $(\mathrm{DN} / \mathrm{s})$ to flux density in milli-Jansky.

\begin{tabular}{|l|l|l|}
\hline Band & ZP (mag) & $\begin{array}{l}\text { Cal Factor } \\
(\mathrm{DN} / \mathrm{s} / \mathrm{mJy})\end{array}$ \\
\hline $\mathrm{J}$ & $21.24+/-0.04$ & 183.074 \\
\hline $\mathrm{H}$ & $20.91+/-0.05$ & 212.974 \\
\hline $\mathrm{K}$ & $19.96+/-0.06$ & 130.563 \\
\hline Paschen- $\alpha$ & $17.50+/-0.09$ & 10.311 \\
\hline Paschen- $\alpha$ cont. & $17.46+/-0.08$ & 10.301 \\
\hline
\end{tabular}

Table 1. Zeropoint (ZP) and calibration factors for several FLITECAM filters commissioned in the FLIPO configuration. Here, the ZP is the magnitude that gives $1 \mathrm{DN} / \mathrm{s}$.

Combining the zeropoints with background measurements, we can estimate the minimum detectable continuum fluxes (for $\mathrm{S} / \mathrm{N}=4$ in $900 \mathrm{~s}$ ) for the various filters. The results are sensitive to the image size, and of course apply only to the FLIPO configuration. For J, H, and $\mathrm{K}$, the limiting fluxes are $20.17,25.21$, and $39.64 \mu \mathrm{Jy}$. For the Paschen- $\alpha$ filters the value is $\sim 135 \mu \mathrm{Jy}$.

Zeropoints were harder to obtain for the thermal infrared filters. Brighter stars were needed, and detector sub-arrays had to be used to read out the array faster in order to avoid saturation. Nevertheless, our preliminary results for Ice, PAH, and $\mathrm{nbL}$ yield the following detection limits in FLIPO configuration: $0.77,1.5$, and $1.4 \mathrm{mJy}$.

Figure 2 shows the transmission profiles for all of the filters commissioned in the FLIPO configuration, including the three $4 \%$ wide filters centered at $3.08 \mu \mathrm{m}$ (Ice), $3.3 \mu \mathrm{m}$ (PAH), and $3.6 \mu \mathrm{m}$ (nbL). The filter profiles are over-plotted on ATRAN atmospheric models ${ }^{12}$ to illustrate the difference in water vapor overburden between Mauna Kea $(14,000 \mathrm{ft})$ and SOFIA $(41,000 \mathrm{ft})$. From the close-up of the region containing Paschen- $\alpha$, it is clear that this emission line is heavily attenuated by the lower atmosphere for ground-based sites, giving FLITECAM an advantage for studying this otherwise unattainable parameter space. During commissioning, the Paschen- $\alpha$, and the Paschen- $\alpha$ continuum filters were used for two quite diverse science projects, the study of a star-formation region and the transit of an exoplanet. 

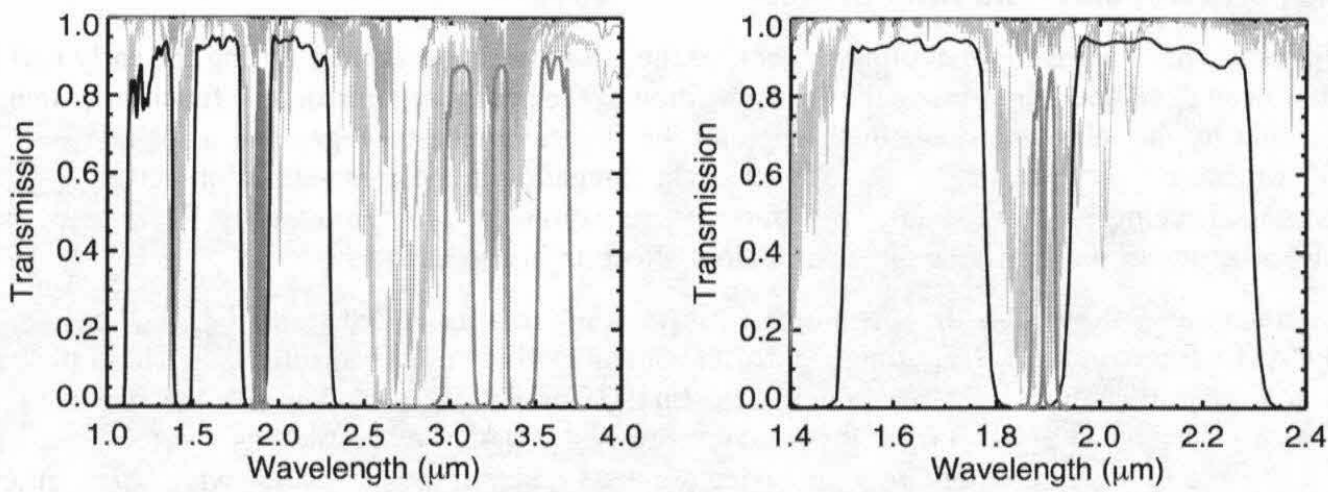

Figure 2. Shows the transmission profiles of FLITECAM filters in the 1-4 $\mu \mathrm{m}$ range compared to ATRAN models of atmospheric transmission for Mauna Kea (14,000 ft; grey) and SOFIA (41,000 ft; yellow). The left hand plot includes three broad band filters (JHK; black) and five narrow band filters (in red from L to R: Paschen- $\alpha$, Paschen- $\alpha$ continuum, $3.08 \mu \mathrm{m}$ Ice, $\mathrm{PAH}$, and $\mathrm{nbL}$ ). The right hand plot is a close-up of the Paschen- $\alpha$ region clearly showing the advantage of observing Paschen- $\alpha$ from the stratosphere.

\subsection{Paschen- $\alpha$ and PAH imaging of NGC2024}

To demonstrate FLITECAM's ability to observe wavelengths typically obscured by water vapor at lower altitudes, as well as its ability to perform imaging of extended objects, the FLITECAM team observed NGC 2024, the Flame Nebula, in Orion. NGC 2024 is a well-known nebula with a dense dust lane that obscures many infrared sources. Figure 3 shows FLITECAM images of the region around the ionizing source ${ }^{13}$ IRS $2 b$, which is partially blended with the brighter IRS 2 source about 5" away.

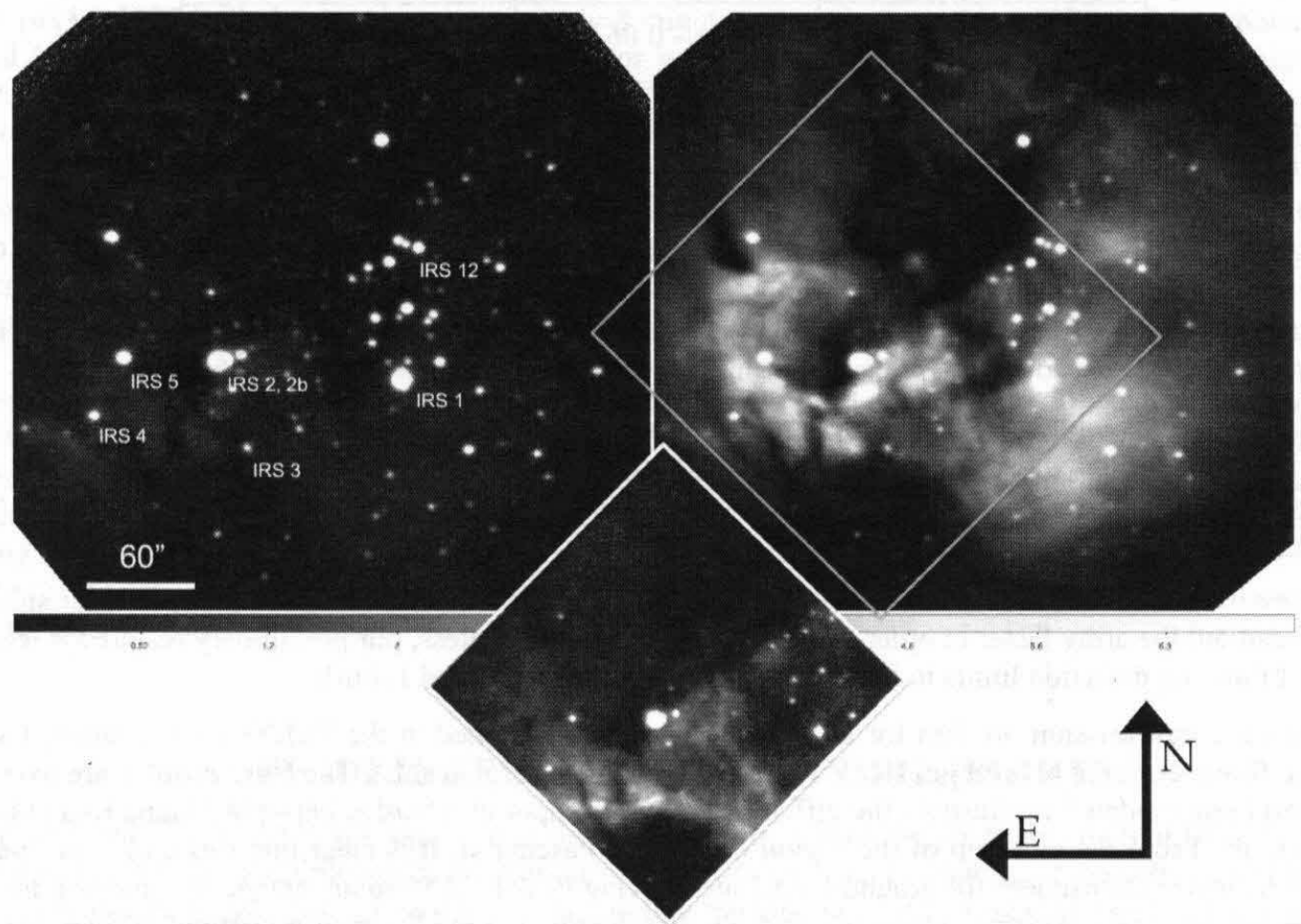

Figure 3. Three FLITECAM images of NGC 2024. Upper left: Paschen- $\alpha$ continuum at $1.90 \mu \mathrm{m}$; Upper right: Paschen- $\alpha$ emission at $1.875 \mu \mathrm{m}$; Below: a $512 \times 512$ pixel sub-array field in the $3.3 \mu \mathrm{m}$ PAH filter. The approximate location of the PAH sub-array field is shown on the Paschen- $\alpha$ image by a green box. Each image was reduced using the

FLITECAM imaging pipeline. 
The images in Figure 3 use three narrow filters; the Paschen- $\alpha$ continuum at $1.90 \mu \mathrm{m}$, the Paschen- $\alpha$ line at $1.875 \mu \mathrm{m}$, and a sub-array field in the $3.3 \mu \mathrm{m}$ PAH filter. The comparison clearly illustrates the dramatic distribution of emission from ionized hydrogen. Moreover, a careful examination of the images reveals that the emission from polycyclic aromatic hydrocarbon (PAH) grains is mainly located on the outer edge of the Paschen- $\alpha$ emission region, and is not coincident with the Paschen- $\alpha$ emission. The PAH image is a $512 \times 512$ sub-array to enable the detector to be read out fast enough to prevent saturation on the background. FLITECAM can be further sub-arrayed to achieve even faster readouts, an advantage of this detector. All sub arrays are fixed around the center of the detector.

\subsection{Spectral coverage}

A major activity for the 2014 commissioning flights was calibration of FLITECAM's nine spectroscopic modes, each of which can be observed with two different resolving powers, as discussed in Section 2. Figure 4 shows the approximate wavelength ranges of the three KRS-5 grisms, labeled A, B, and C, each of which can be used in three different orders with a matching blocking filter in the first filter wheel. FLITECAM has nearly continuous coverage from 1-5.5 $\mu \mathrm{m}$.

Though most commissioning spectra were taken using the lower resolution slit, $\mathrm{R} \sim 1300$, boresight reference points on both slits were established. We demonstrated successfully that we could both nod the star back and forth along the slit (in the typical ' $A B$ ' nod pattern) and 'on/off' the slit in instrument coordinates. Because of the increased thermal background in FLIPO configuration, the longest wavelength regime of Grism A has not been commissioned. Wavelength calibrations are available for all of the other combinations.

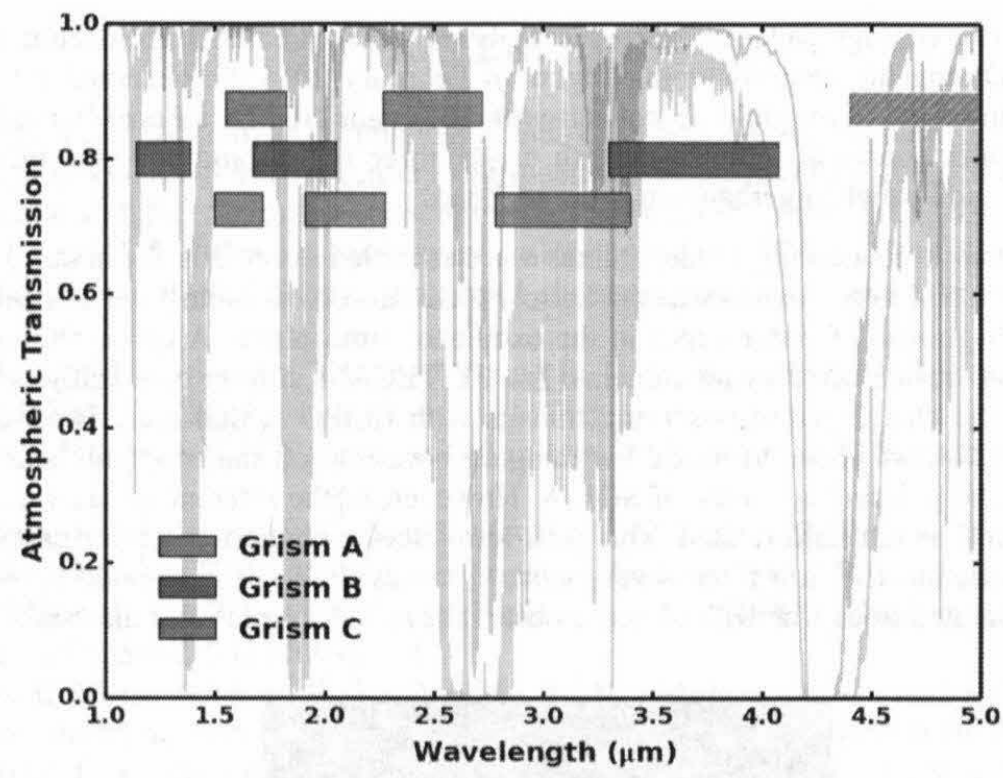

Figure 4. Spectral coverage of the three FLITECAM grisms, labeled A, B, and C. Each grism can be used in three orders by selecting a suitable order-sorting filter from filter wheel \#1. For comparison, the ATRAN models of atmospheric transmission are shown for Mauna Kea (red) and the stratosphere (blue). The longest wavelength regime of Grism A (red hashed rectangle) has not been commissioned.

\subsection{Spectroscopy of SN 2014J}

During the February 2014 flights, the most significant application of the spectroscopic mode was observations of the Type Ia supernova in M82 (SN 2014J). Figure 5 shows the preliminary accumulated spectra of the supernova. Also shown in Figure 5 is a picture of the FLIPO team as we maneuvered the supernova onto the FLITECAM slit.

The blue shaded regions in Figure 5 indicate parts of the spectra that are very difficult to observe from the ground because of low atmospheric transmission. We also have data for the 2.7-3.5 $\mu \mathrm{m}$ regime which are currently being analyzed. All spectra were reduced using a version of the SpeXtool package ${ }^{14,15}$ modified for use with FLITECAM. Note that the supernova brightened over the course of one week. 

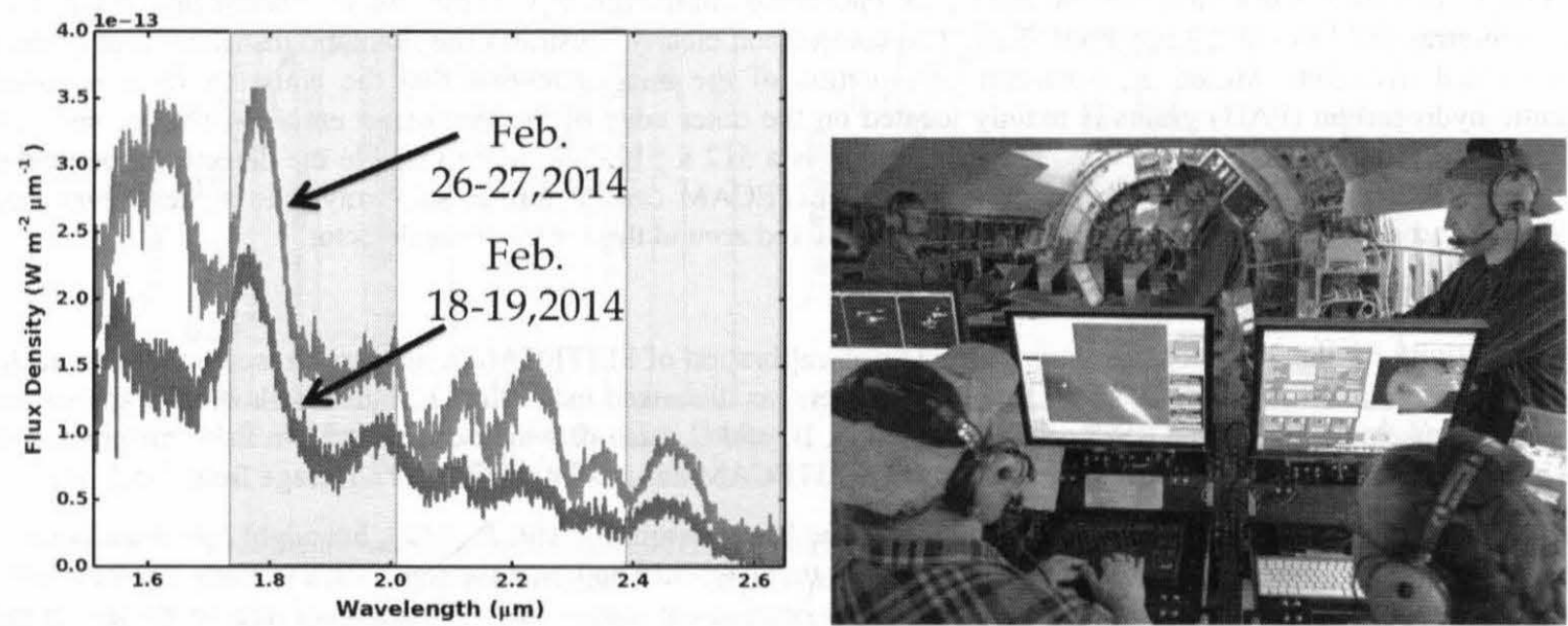

Figure 5. Spectra of SN 2014J. Four of FLITECAM's grism modes were used to stitch together this spectrum. Note the brightening of the features with time. The picture shows the FLIPO team in flight as M82 is moved onto the slit.

\subsection{Transit photometry of GJ1214b}

Simultaneous optical and infrared, high cadence photometric observations of transits and occultations is a major goal of the FLIPO configuration. During the commissioning flights in February 2014 we observed a transit of the exoplanet GJ1214b. Figure 6 shows an image of the field. In concert with HIPO and SOFIA's Focal Plane Imager (FPI), we were able to image a transit simultaneously in four wavelength bands, three optical and one near-infrared. The PIs for this observation, Claudia Dryer and Daniel Angerhaussen, were on board.

Using FLITECAM's continuous data-taking mode, frames were recorded every $30 \mathrm{~s}$ for about 3.5 hours. The infrared band selected for the FLITECAM observations was the narrow Paschen- $\alpha$ continuum filter at $1.90 \mu \mathrm{m}$. This wavelength was chosen as a possible diagnostic of water vapor in the exoplanet atmosphere. A quick photometric analysis during flight showed that the transit was detected by all cameras. For FLITECAM, further data analysis is needed to eliminate systematic effects. One of the challenges of observing transits with FLIPO is that there is no compensation for field rotation. In the case of GJ1214, we chose to locate the imaging boresight on the exoplanet star so that the target star would remain fixed within the pointing accuracy of SOFIA. However, as the reference stars were not on the boresight, they moved to different pixels as the field rotated. This drift introduces a photometric error unless the flat field is very good and very stable. Because the $1.90 \mu \mathrm{m}$ filter is very narrow, it was challenging to build up enough signal for a flat field using the sky. We attempted to do this with dithered observations before and after the transit. Further analysis is in progress.

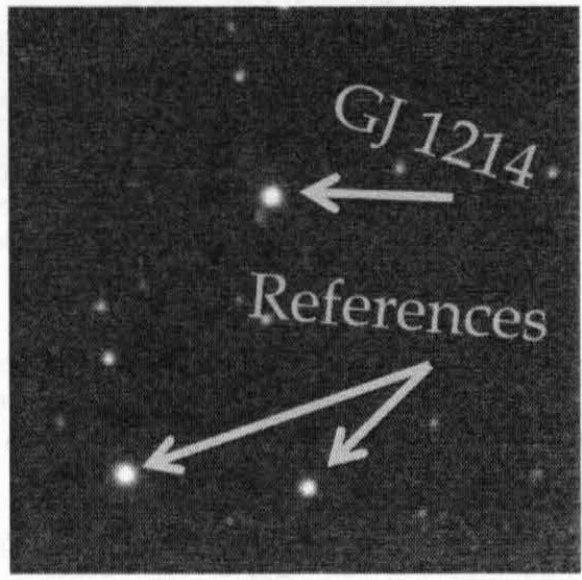

Figure 6. Image of the field containing GJ 1214. This frame was based on a set of five-point dither images taken on a test flight leg a few days before the actual transit observations. The filter was the $1 \%$ wide filter at $1.90 \mu \mathrm{m}$. 


\section{CONCLUSIONS}

Several improvements were made to both FLITECAM hardware and software following the observatory verification flights in October 2011. During six flights in February 2014, FLITECAM was commissioned successfully in the FLIPO configuration. This configuration has FLITECAM co-mounted with the optical instrument, HIPO. In this arrangement there is a warm dichroic beam splitter and a fold mirror in front of the FLITECAM entrance window. The emissivity and increased background made it impractical to commission FLITECAM beyond $4 \mu \mathrm{m}$. We have shown that FLITECAM, in the FLIPO configuration, is suitable for narrow band Paschen- $\alpha$ and PAH imaging, which is difficult from the ground, as well as spectroscopy from 1-4 $\mu \mathrm{m}$. Preliminary analysis of an exoplanet transit is encouraging for high cadence photometric observations, such as transits and occultations. Finally, we were fortunate that a target of opportunity occurred during our flight series, supernova SN 2014J. The FLITECAM spectroscopic observations provided data coverage for spectral regions that are not well transmitted to the ground.

\section{ACKNOWLEDGMENTS}

The authors wish to acknowledge the hard work and dedication of the current FLITECAM project team at UCLA, including Ted Aliado, George Brims, and Ken Magnone, We also thank the entire flight support team at DAOF and USRA. FLITECAM is funded primarily by a grant from USRA.

\section{REFERENCES}

[1] McLean, I. S., Smith, E. C., Aliado, T., Brims, G., Kress, E., Magnone, K., Milburn, J., Oldag, A., Silvers, T., and Skulason, G., "FLITECAM, a 1-5 micron camera and spectrometer for SOFIA," Proc. SPIE, 6269, 195 (2006).

[2] Smith, E. C. and McLean, I. S., "Grism spectroscopy with FLITECAM,” Proc. SPIE, 6269, 56 (2006).

[3] Smith, E. C. and McLean, I. S., "Ground-based commissioning of FLITECAM," Proc SPIE 7014, 701411 (2008).

[4] Mainzer, A. K. and McLean, I. S., "Using narrowband photometry to detect young brown dwarfs in IC 348," ApJ, 597, 555 (2003).

[5] Mainzer, A. K., McLean, I. S., Sievers, J. L., and Young E. T., "Using narrowband photometry to classify stars and brown dwarfs," ApJ, 604, 832 (2004).

[6] Smith, E. C. D. and McLean, I. S., “A survey of 3.3 micron PAH emission in Planetary Nebulae,” ApJ, 676, 408415 (2008).

[7] Dunham, E. W., Bida, T. A., Collins, P. L., Mandushev, G. I., McLean, I. S., Person, M. J., Smith, E. C., Taylor, B. W., and Zoonematkermani, S., "HIPO in-flight performance aboard SOFIA," Proc. SPIE, 8446, 884618-15 (2012).

[8] Dunham, E. W., Elliot, J. L., Bida, T. A., and Taylor, B. W., "HIPO: a high-speed imaging photometer for occultations," Proc. SPIE, 5492, 592 (2004).

[9] McLean, I. S., Smith, E. C., Becklin, E. E., Dunham, E. W., Milburn, J. W., and Savage, M. L, "FLITECAM: current status and results from observatory verification flights," Proc. SPIE, 8446, 844618-15 (2012).

[10] Dunham, E. W., Bida, T. A., Collins, P. L., Mandushev, G., Zoonematkermani, S., Van Cleve, J., Angerhausen, D., and Mandell, A., "HIPO in-flight performance improvements," Proc. SPIE, 9147, paper 16. (2014).

[11] Cohen, M., Megeath, S.T., Hammersley, P.L., Martin-Luis, F., and Stauffer, J., "Spectral irradiance calibration in the infrared. XIII. 'Supertemplates' and on-orbit calibrators for the SIRTF array camera," AJ, 125, 2645 (2003).

[12] Lord, S. D. "A New Software Tool for Computing Earth's Atmospheric Transmission of Near- and Far-Infrared Radiation," NASA Technical Memorandum 103957 (1992).

[13] Bik, A., Lenorzer, A., Kaper, L., Comeron, F., Waters, L. B. F. M., de Koter, A., and Hanson, M. M., "Identification of the ionizing source of NGC 2024," A\&A, 404, 249 (2003).

[14] Cushing, M. C., Vacca, W. D., and Rayner, J. T., "Spextool: A Spectral Extraction Package for SpeX, a 0.8-5.5 Micron Cross-Dispersed Spectrograph," PASP, 116, 362 (2004).

[15] Vacca, W. D., Cushing, M. C., and Rayner, J., T., "A Method of Correcting Near-Infrared Spectra for Telluric Absorption," PASP, 115, 805 (2003). 